\title{
DIFFERENTIAL FORMS AND LIE ALGEBRA COHOMOLOGY FOR ALGEBRAIC LINEAR GROUPS
}

\author{
BY \\ G. Hochschild and B. Kostant
}

\section{Introduction}

In the study of the rational cohomology theory of algebraic linear groups, the differential forms, constructed from the algebra of the rational representative functions on the group, play a major role in providing the link between the group cohomology and the Lie algebra cohomology [5]. Moreover, the cohomology of the differential forms has some significance as an algebraic geometric invariant. For instance, it follows from [5, Theorem 4.1] that, if $R$ is the algebra of the rational representative functions on an irreducible algebraic linear group $G$ over a field $F$ of characteristic 0 , the cohomology of the differential forms of $R$ is trivial (if and) only if $R$ is an ordinary polynomial algebra over $F$.

Our main purpose here is to extend the theory of these differential forms to the case where the group $G$ is replaced by a "homogeneous space" $G / K$, $K$ being a fully reducible algebraic subgroup of $G$. This means that $R$ is replaced by the subalgebra $R^{K}$ of $R$ consisting of the functions that are constant on the cosets of $K$ in $G$. If the base field $F$ is algebraically closed, the designation of $G / K$ as a homogeneous space is actually justified: we shall see (Theorem 5.1) that $G / K$ has then the structure of an affine algebraic variety, with $R^{K}$ as its algebra of polynomial functions. The connections between the cohomology of the differential forms, the rational cohomology of the group, and the Lie algebra cohomology extend to this case, with the relative Lie algebra cohomology taking the place of the ordinary Lie algebra cohomology (Theorems 3.1, 3.2, 3.3, 4.2). In order to get the full information here, it was necessary to extend the known tensor product decomposition theory for Lie algebra cohomology to the relative case (Section 4), and this may be of independent interest.

For an arbitrary unitary $F$-algebra $P$, there are two known constructions giving a complex of "differential forms." $O$ One of these is based on the $F$-derivations of $P$ (Section 3 ), while the other is quite direct and purely formal (Section 5). We show that, for $P=R^{K}$, the two complexes thus obtained are naturally isomorphic (Theorem 5.2). Actually, this result holds under more general circumstances; results of Kunz [6] are relevant here.

We take this opportunity to thank M. Rosenlicht for his help in some clarifying discussions on the topic of differential forms.

\section{The algebra of the rational representative functions}

Let $G$ be an irreducible algebraic linear group over an infinite field $F$. We denote by $R(G)$, or simply by $R$, the $F$-algebra of the rational representative

Received February 17, 1961. 
functions on $G$, i.e., of the $F$-valued rational functions defined at every point of $G$ and with the property that their translates under the action of $G$ span only a finite-dimensional space of functions. Equivalently, the rational representative functions are those rational functions which remain everywhere defined under arbitrary extensions of the base field $F$. If $G$ is given concretely as an algebraic group of linear transformations of determinant 1 , then $R$ coincides with the algebra of all polynomial functions on $G$. The field of quotients, $Q$, of $R$ is the field of the rational functions on $G$.

We consider the action of $G$ by left translations on $R$ and on $Q$; for $f \in R$, and $x$ and $y$ in $G$, the left translate $x \cdot f$ of $f$ is defined by $(x \cdot f)(y)=f(y x)$. Let (5) denote the Lie algebra of $G$. The action of $G$ on $R$ by left translations induces an action of $\$$ ) on $R$ and hence on $Q$ by which $\$$ acts as a Lie algebra of $F$-derivations. In this way, \& becomes identified with the Lie algebra of all $F$-derivations of $R$ (or of $Q$, by canonical extension of derivations) that commute with the right translations $f \rightarrow f \cdot x$, where $(f \cdot x)(y)=f(x y)$. The $Q$-space of all $F$-derivations of $Q$ is spanned by $\&$ and in fact is canonically isomorphic with $Q \otimes(5)$. Moreover, the $R$-module of all $F$-derivations of $R$ is canonically isomorphic with $R \otimes$ \& (see [5, Lemma 4.1], and [1]).

Lemma 2.1. Let $K$ be an algebraic subgroup of the irreducible algebraic linear group $G$, and let $Q^{K}$ denote the subfield of $Q$ consisting of the elements left fixed by the left translations from $K$. Then $Q^{K}$ separates the cosets $x K$ of $K$ in $G$. Furthermore, every element of (5) that annihilates $Q^{K}$ belongs to the Lie algebra $\Omega$ of $K$.

Proof. Let $I$ denote the ideal of $R$ that is associated with $K$. We can evidently find $F$-linearly independent elements $f_{1}, \cdots, f_{n}$ of $I$ such that $I=R f_{1}+\cdots+R f_{n}$ and $F f_{1}+\cdots+F f_{n}$ is stable under the action of $K$ by left translations. Let $V$ denote the smallest $G$-submodule of $R$ (under the action of $G$ by left translations) that contains all the $f_{i}$ 's. Let $W$ be the homogeneous component of degree $n$ of the exterior $F$-algebra built over $V$. We consider the action of $G$ and \&5 on $W$ that is induced by the action of $G$ on $V$. Let $w$ be the exterior product, in $W$, of $f_{1}, \cdots, f_{n}$. Then $F w$ is evidently a $K$-stable 1-dimensional subspace of $W$. If $\gamma$ is any element of the dual space to $W$, we define the element $\gamma / w$ of $R$ by $(\gamma / w)(x)=\gamma(x \cdot w)$, for every $x \in G$. If $\alpha$ and $\beta$ are elements of the dual space to $W$, and if $\beta / w \neq 0$, the quotient $(\alpha / w) /(\beta / w)$ is immediately seen to be an element of $Q^{K}$, as a consequence of the fact that $F w$ is $K$-stable. Now let $y$ be an element of $G$ that does not belong to $K$. Then $y \cdot w \notin F w$, because otherwise $y \cdot I \subset I$, which implies that $y \in K$. Hence we can choose $\alpha$ and $\beta$ from the dual space to $W$ such that $\alpha(w)=1, \alpha(y \cdot w)=0, \beta(w)=1, \beta(y \cdot w)=1$. Then $(\alpha / w) /(\beta / w)$ is defined at the points 1 and $y$ of $G$ and takes the values 1 and 0 at these points, respectively. Thus $Q^{K}$ separates the cosets $y K$ and $K$, which proves the first part of Lemma 2.1.

Now let $\zeta$ be an element of $\$$ that does not belong to $\Re$. Then $\zeta(w) \notin F w$, because otherwise $\zeta(I) \subset I$ which implies that $\zeta \in \Omega$. Hence we can choose 
$\alpha$ and $\beta$ from the dual space to $W$ such that $\alpha(w)=1, \alpha(\zeta(w))=0, \beta(w)=1$, $\beta(\zeta(w))=1$. The transform of $(\alpha / w) /(\beta / w)$ by $\zeta$ is

$$
\left((\alpha / \zeta(w))(\beta / w)-(\alpha / w)(\beta(\zeta(w))) /(\beta / w)^{2} \neq 0 .\right.
$$

This establishes the second part of Lemma 2.1.

LEMma 2.2. The transcendence degree of $Q$ over $Q^{K}$ is equal to the dimension of $K$.

Proof. $Q$ is a finitely generated separable extension of $Q^{K}$, whence the transcendence degree of $Q$ over $Q^{K}$ is equal to the dimension of the $Q$-space of the $Q^{K}$-derivations of $Q$. This space contains the canonical isomorphic image of $Q \otimes \Omega$ whose dimension is equal to the dimension of $\Omega$ over $F$, which is equal to the dimension of $K$. Hence it suffices to show that every $Q^{K}$-derivation $\tau$ of $Q$ belongs to the canonical image of $Q \otimes \Re$. Like every $F$-derivation of $Q, \tau$ is of the form $\sum_{i} q_{i} \zeta_{i}$, with $q_{i} \in Q$ and $\zeta_{i} \in$ \$S. Subtracting an element of $Q \Omega$ and multiplying by a nonzero element of $R$, we obtain a $Q^{K}$ derivation of $Q$ that has the form $\sum_{i} f_{i} \xi_{i}$, where the $f_{i}$ 's are elements of $R$ and the $\zeta_{i}$ 's are elements of (5) that are linearly independent mod $\Omega$. Now what we have to show is that the $f_{i}$ 's are 0 . We have $\sum_{i} f_{i} \zeta_{i}(q)=0$, for every $q \in Q^{K}$. Translating from the right with an arbitrary element $x$ of $G$, and noting that $Q^{K} \cdot x=Q^{K}$, we find that $\sum_{i}\left(f_{i} \cdot x\right) \zeta_{i}(q)=0$, for every $q \in Q^{K}$. Let $q \in Q^{K}$, and let $y$ be an element of $G$ at which each $\zeta_{i}(q)$ is defined. Let $z$ be an arbitrary element of $G$. Then we have

$$
\sum_{i} f_{i}(z) \zeta_{i}(q)(y)=\left(\sum_{i}\left(f_{i} \cdot z y^{-1}\right) \zeta_{i}(q)\right)(y)=0
$$

Thus we may conclude that $\sum_{i} f_{i}(z) \zeta_{i}(q)=0$, for every $q \epsilon Q^{K}$. By the second part of Lemma 2.1, this implies that $\sum_{i} f_{i}(z) \zeta_{i} \epsilon \Re$, whence each $f_{i}(z)=0$. This completes the proof of Lemma 2.2 .

Proposition 2.1. Let $G$ be an irreducible algebraic linear group over the infinite field $F$, and let $K$ be an algebraic subgroup of $G$. If $K$ is unipotent, or if $F$ is of characteristic 0 and $K$ is fully reducible, then the field of quotients of $R^{K}$ coincides with $Q^{K}$.

Proof. We must show that, if $q$ is any nonzero element of $Q^{K}$,

$$
(R q \cap R)^{K} \neq 0 \text {. }
$$

This is evidently the case if $K$ is unipotent, because in that case every element of $R$ generates a unipotent $K$-module.

Now suppose that $F$ is of characteristic 0 and $K$ is fully reducible. Let $V$ be a nonzero simple $K$-submodule of $R q \cap R$. Let $V^{\prime}$ be the dual $K$-module $\operatorname{Hom}_{F}(V, F)$. Let $S$ be the space of the representative functions on $K$ that are associated with $V^{\prime}$. There is a $K$-module monomorphism of $V^{\prime}$ into the direct sum of a finite number of copies of $S$. Since $V$ is simple, so is $V^{\prime}$, and we may conclude that $V^{\prime}$ is isomorphic, as a $K$-module, with a submodule 
of $S$. Now observe that the restriction of functions on $G$ to $K$ yields a $K$-module epimorphism of $R(G)$ onto $R(K)$. Since $K$ is fully reducible and $F$ is of characteristic $0, R(G)$ is semisimple as a $K$-module. Hence there is a $K$-module monomorphism $R(K) \rightarrow R(G)$ that is inverse to the restriction map. Composing this with a $K$-module monomorphism $V^{\prime} \rightarrow S$, we obtain a $K$-module monomorphism $\varphi$ of $V^{\prime}$ into $R$. Now let $v_{1}, \cdots, v_{n}$ be a basis for $V$, and let $f_{1}, \cdots, f_{n}$ be the dual basis for $V^{\prime}$. We choose the $v_{i}$ so that $v_{1}(1)=1$, while $v_{i}(1)=0$, for every $i>1$. Put $g_{i}=\varphi\left(f_{i}\right)$. Then, for every $x \in G$, we have $\sum_{i=1}^{n}\left(g_{i} \cdot x\right) v_{i} \epsilon(R q \cap R)^{K}$. Choosing $x$ so that $g_{1}(x) \neq 0$, we ensure that this function is not 0 . This completes the proof of Proposition 2.1.

Proposition 2.2. Under the assumptions of Proposition 2.1, every F-derivation of $R^{K}$ into $R$ extends to an $F$-derivation of $R$.

Proof. Let $\tau$ be an $F$-derivation of $R^{K}$ into $R$. Then $\tau$ extends canonically to an $F$-derivation of the field of quotients of $R^{K}$ into $Q$, i.e., by Proposition 2.1, to an $F$-derivation of $Q^{K}$ into $Q$. This, in turn, can be extended to an $F$-derivation of $Q$, because $Q$ is separable over $Q^{K}$. Let $\zeta_{1}, \cdots, \zeta_{s}$ be a basis for $\Omega$, and extend this to a basis $\zeta_{1}, \cdots, \zeta_{s+t}$ for $\$$. There is an $F$-derivation of $Q$ extending $\tau$ that has the form $\sum_{i=1}^{t} q_{i} \zeta_{s+i}$, with $q_{i} \in Q$. By Lemma 2.2, the transcendence degree of $Q^{K}$ over $F$ is equal to $t$. Since $Q$ is finitely generated and separable over $F$, so is $Q^{K}$, and every finite system of generators for $Q^{K}$ over $F$ contains a separating transcendence base for $Q^{K}$ over $F$. Hence there are elements $u_{1}, \cdots, u_{t}$ in $R^{K}$ that form a separating transcendence base for $Q^{K}$ over $F$. Since $Q$ is separable over $Q^{K}$, we can complete this to a separating transcendence base $u_{1}, \cdots, u_{t+s}$ for $Q$ over $F$. Then no nonzero $F$-derivation of $Q$ annihilates each $u_{i}$, and it follows that the determinant formed with the $\zeta_{j}\left(u_{i}\right)$ is different from 0 . On the other hand, $\zeta_{j}\left(u_{i}\right)=0$ whenever both $i \leqq t$ and $j \leqq s$. Hence the determinant formed with the $\zeta_{s+k}\left(u_{i}\right)$, where $i$ and $k$ range from 1 to $t$, is not equal to 0 . Let $D$ denote this determinant. Now we have

$$
\sum_{k=1}^{t} q_{k} \zeta_{s+k}\left(u_{i}\right)=\tau\left(u_{i}\right) \epsilon R, \quad \text { for each } i \leqq t .
$$

Hence $D q_{k} \in R$, for each $k$. Let $J$ denote the ideal of all $f \in R$ such that $f q_{k} \epsilon R$, for each $k$. We have just shown that $D \in J$. On the other hand, we may evidently replace $u_{1}, \cdots, u_{t}$ by $u_{1} \cdot x, \cdots, u_{t} \cdot x$, where $x$ is an arbitrary element of $G$. This replaces $D$ by $D \cdot x$, so that we conclude that $D \cdot x \in J$, for every $x \in G$. Hence $J$ has no zero on $G$.

If $F$ is algebraically closed, every proper ideal of $R$ lies in the kernel of some $F$-homomorphism $R \rightarrow F$. On the other hand, every such homomorphism is of the form $f \rightarrow f(x)$, with some $x \in G$. Hence we conclude that, if $F$ is algebraically closed, $J=R$, i.e., $q_{k} \in R$, for each $k$, so that our extended $\tau$ actually sends $R$ into $R$. In the general case, we extend $G$ and $K$ canonically over the algebraic closure $F^{*}$ of $F$; let $G^{F^{*}}, K^{F^{*}}$ denote these extended groups. 
Then we have

$$
R\left(G^{F^{*}}\right)=R(G) \otimes F^{*} \text { and }\left(R\left(G^{F^{*}}\right)\right)^{K^{F^{*}}}=R(G)^{K} \otimes F^{*} .
$$

Hence it is clear that Proposition 2.2 for $\left(G^{F^{*}}, K^{F^{*}}\right)$ implies Proposition 2.2 for $(G, K)$. This completes the proof.

Observe that Proposition 2.2 implies that the canonical map of $R \otimes(\$ / \Re)$ into the $R$-module of the $F$-derivations of $R^{K}$ into $R$ is an epimorphism. The argument we made at the end of the proof of Lemma 2.2 shows that this map is also a monomorphism. Thus the $R$-module of all $F$-derivations of $R^{K}$ into $R$ is canonically isomorphic with $R \otimes(\$ / \Omega)$. Regard $\$ / \Omega$ as a $K$-module via the adjoint representation of $G$ on $(\$)$, and regard $R \otimes(\$) / \Omega)$ as the tensor product of the $K$-modules $R$ (by left translation) and (\$) $\Omega$. Then we have the following result.

CoRollary 2.1. Under the assumptions of Proposition 2.2, the $R^{K}$-module of all $F$-derivations of $R^{K}$ is canonically isomorphic with $\left(R \otimes((5 / \Omega))^{K}\right.$.

Proof. Let $\sum_{i} f_{i} \otimes \zeta_{i}$ be an element of $\left.R \otimes(\$) / \Omega\right)$, where $f_{i} \in R$ and $\zeta_{i} \in(\$)$. Let $g \in R^{K}$. Then we have, for every $x \in K$,

$$
\begin{aligned}
x \cdot\left(\sum_{i} f_{i} \zeta_{i}(g)\right)=\sum_{i}\left(x \cdot f_{i}\right) x \cdot \zeta_{i}(g) & \\
& =\sum_{i}\left(x \cdot f_{i}\right)\left(x \cdot \zeta_{i}\right)(x \cdot g)=\sum_{i}\left(x \cdot f_{i}\right)\left(x \cdot \zeta_{i}\right)(g) .
\end{aligned}
$$

Hence our derivation sends $R^{K}$ into $R^{K}$ if and only if it coincides with the derivation of $R^{K}$ effected by $x \cdot\left(\sum_{i} f_{i} \otimes \zeta_{i}\right)$, for every $x \in K$. By the remark that just precedes the statement of our corollary, this is so if and only if $\sum_{i} f_{i} \otimes \zeta_{i} \epsilon(R \otimes(\$ / \Re))^{K}$, Q.E.D.

\section{The complex of differential forms}

Let $P$ be a commutative unitary $F$-algebra, where $F$ is a field. Let $T_{P}$ denote the $P$-module of all $F$-derivations of $P$. For every positive integer $q$, let $A^{q}\left(T_{P}\right)$ denote the $P$-module of all $(P, q)$-multilinear alternating maps from $T_{P}$ to $P$, and put $A^{0}\left(T_{P}\right)=P$. The elements of $A^{q}\left(T_{P}\right)$ are called the homogeneous differential forms of degree $q$ on $P$. The weak direct sum of the $A^{q}\left(T_{P}\right)$ will be denoted $A\left(T_{P}\right)$. With every $\tau \in T_{P}$, we associate a $P$-linear homogeneous endomorphism of degree -1 of $A\left(T_{P}\right)$, called the contraction with respect to $\tau$ and denoted $c_{\tau}$. This is defined as follows: $c_{\tau}=0$ on $P$; for $\alpha \epsilon A^{q}\left(T_{P}\right)$ and $q>0, c_{\tau}(\alpha)$ is the element of $A^{q-1}\left(T_{P}\right)$ given by

$$
c_{\tau}(\alpha)\left(\tau_{1}, \cdots, \tau_{q-1}\right)=\alpha\left(\tau, \tau_{1}, \cdots, \tau_{q-1}\right) .
$$

The natural action of $T_{P}$ on $P$ is extended to an action of $T_{P}$ on $A\left(T_{P}\right)$ by homogeneous $F$-linear endomorphisms $t_{\tau}$ of degree 0 , where $t_{\tau}(\alpha)\left(\tau_{1}, \cdots, \tau_{q}\right)=\tau\left(\alpha\left(\tau_{1}, \cdots, \tau_{q}\right)\right)+\sum_{i=1}^{q} \alpha\left(\tau_{1}, \cdots,\left[\tau_{i}, \tau\right], \cdots, \tau_{q}\right)$.

One verifies directly that $c_{\tau}^{2}=0,\left[t_{\tau}, c_{\sigma}\right]=c_{[\tau, \sigma]}$, and $\left[t_{\tau}, t_{\sigma}\right]=t_{[\tau, \sigma]}$. Next 
one verifies inductively on the degree that there is one and only one homogeneous $F$-linear endomorphism $\delta$ of degree 1 on $A\left(T_{P}\right)$ satisfying $\delta c_{\tau}+c_{\tau} \delta=t_{\tau}$, for all $\tau \in T_{P}$. Then $\delta$ commutes with each $t_{\tau}$ and $\delta^{2}=0$. One has the familiar explicit formula for $\delta$ :

$$
\begin{aligned}
(\delta \alpha)\left(\tau_{0}, \cdots, \tau_{q}\right) & =\sum_{i=0}^{q}(-1)^{i} \tau_{i}\left(\alpha\left(\tau_{0}, \cdots, \hat{\tau}_{i}, \cdots, \tau_{q}\right)\right) \\
& +\sum_{r<s}(-1)^{r+s} \alpha\left(\left[\tau_{r}, \tau_{s}\right], \tau_{0}, \cdots, \hat{\tau}_{r}, \cdots, \hat{\tau}_{s}, \cdots, \tau_{q}\right)
\end{aligned}
$$

The complex $\left(A\left(T_{P}\right), \delta\right)$ is called the complex of the differential forms on $P$.

We shall examine this complex in the cases $P=R$ and $P=R^{K}$. The case $P=R$ was investigated in [5], and we shall obtain results on $R^{K}$ from a certain canonical map of the complex for $R^{K}$ into the complex for $R$.

We identify $T_{R}$ with $R \otimes$ (5, and we define an $F$-linear projection $\beta: T_{R} \rightarrow$ (S) by $\beta\left(\sum_{i} f_{i} \otimes \zeta_{i}\right)=\sum_{i} f_{i}(1) \zeta_{i}$. For every $\zeta \epsilon$ (\$), we define the derivation $\zeta^{*}$ of $R$ by $\zeta^{*}(f)(x)=\zeta(x \cdot f)(1)$, so that $\zeta^{*}$ commutes with the left translations by the elements of $G$ and $\zeta^{*}(f)(1)=\zeta(f)(1)$, for every $f \epsilon R$. Clearly, $\zeta^{*}\left(R^{K}\right) \subset R^{K}$, and we make the convention that where $\zeta^{*}$ occurs as an argument with a differential form on $R^{K}$ it should be replaced with its restriction to $R^{K}$. The adjoint action of $G$ on $(S)$ is extended to the action $\tau \rightarrow x \cdot \tau$ of $G$ on $T_{R}$, where $(x \cdot \tau)(f)=x \cdot \tau\left(x^{-1} \cdot f\right)$, for every $f \in R$. Then we have $\beta(x \cdot(f \tau))=f(x) \beta(x \cdot \tau)$.

Now let $\alpha \in A^{q}\left(T_{R^{K}}\right)$. Then we define a function $\rho(\alpha)$ on $q$-tuples of elements of $T_{R}$ and with values in $R$ by setting

$$
\rho(\alpha)\left(\tau_{1}, \cdots, \tau_{q}\right)(x)=\alpha\left(\beta\left(x \cdot \tau_{1}\right)^{*}, \cdots, \beta\left(x \cdot \tau_{q}\right)^{*}\right)(x) .
$$

Our last remark shows that $\rho(\alpha)$ is $R$-multilinear, and hence one sees immediately that $\rho(\alpha) \epsilon A^{q}\left(T_{R}\right)$, so that we have defined a homogeneous $R^{K}$-linear map $\rho$ of degree 0 of $A\left(T_{R^{K}}\right)$ into $A\left(T_{R}\right)$.

We claim that $\rho$ commutes with $\delta$. Since the derivations $\zeta^{*}$, with $\zeta$ ranging over (\$), span $T_{R}$ over $R$, it suffices to show that

$$
(\delta \rho \alpha)\left(\zeta_{0}^{*}, \cdots, \zeta_{q}^{*}\right)=(\rho \delta \alpha)\left(\zeta_{0}^{*}, \cdots, \zeta_{q}^{*}\right),
$$

for all $\zeta_{i} \epsilon$ (\$). Let $\zeta \epsilon$ (5). Clearly, $x \cdot \zeta^{*}=\zeta^{*}$, for every $x \in G$. Furthermore, we have $\beta\left(\zeta^{*}\right)=\zeta$. In order to see this, write $\zeta^{*}=\sum_{i} f_{i} \zeta_{i}$, with $f_{i} \in R$ and $\zeta_{i} \epsilon$ (5). Let $f \in R$ and $x \in G$. Then we have

$$
\begin{aligned}
\zeta(f)(x) & =\zeta(f \cdot x)(1)=\zeta^{*}(f \cdot x)(1)=\sum_{i} f_{i}(1) \zeta_{i}(f \cdot x)(1)=\beta\left(\zeta^{*}\right)(f \cdot x)(1) \\
& =\beta\left(\zeta^{*}\right)(f)(x),
\end{aligned}
$$

which proves our assertion.

Hence we have $(\rho \alpha)\left(\zeta_{1}^{*}, \cdots, \zeta_{q}^{*}\right)=\alpha\left(\zeta_{1}^{*}, \cdots, \zeta_{q}^{*}\right)$, and the similar equality for $\delta \alpha$ in the place of $\alpha$. Now the equality to be proved follows at once from the explicit formula for $\delta$, noting that $\left[\zeta_{r}^{*}, \zeta_{s}^{*}\right]=\left[\zeta_{s}, \zeta_{r}\right]^{*}$.

The group $G$ operates on $T_{R}$ and on $T_{R^{K}}$ on the right, as follows:

$$
(\tau \cdot x)(f)=\tau\left(f \cdot x^{-1}\right) \cdot x .
$$


We make $A^{q}\left(T_{R}\right)$ and $A^{q}\left(T_{R} R\right)$ into $G$-modules, setting

$$
(x \cdot \alpha)\left(\tau_{1}, \cdots, \tau_{q}\right)=\alpha\left(\tau_{1} \cdot x, \cdots, \tau_{q} \cdot x\right) \cdot x^{-1} .
$$

We shall show that $\rho$ is a G-module homomorphism. We have

$$
\begin{aligned}
(x \cdot \rho(\alpha))\left(\tau_{1}, \cdots, \tau_{q}\right)(y) & =\rho(\alpha)\left(\tau_{1} \cdot x, \cdots, \tau_{q} \cdot x\right)\left(x^{-1} y\right) \\
& =\alpha\left(\beta\left(x^{-1} y \cdot \tau_{1} \cdot x\right)^{*}, \cdots, \beta\left(x^{-1} y \cdot \tau_{q} \cdot x\right)^{*}\right)\left(x^{-1} y\right) .
\end{aligned}
$$

Now one verifies directly that $\beta\left(x^{-1} \cdot \tau \cdot x\right)=x^{-1} \cdot \beta(\tau)$ and, for $\zeta \in \mathbb{S}$, $\left(x^{-1} \cdot \zeta\right)^{*}=\zeta^{*} \cdot x$. Hence the last expression above is equal to $\alpha\left(\beta\left(y \cdot \tau_{1}\right)^{*} \cdot x, \cdots, \beta\left(y \cdot \tau_{q}\right)^{*} \cdot x\right)\left(x^{-1} y\right)=\rho(x \cdot \alpha)\left(\tau_{1}, \cdots, \tau_{q}\right)(y)$, Q.E.D.

It is seen immediately from the explicit formula that the coboundary operator $\delta$ is also a G-module homomorphism. It is clear that the $G$-module structure just defined on $A\left(T_{R}\right)$ and $A\left(T_{R^{K}}\right)$ is that of a rational $G$-module, in the sense of [5]. Hence it induces the structure of a \$5-module on our complexes, and it is easily verified that this induced (5-module structure coincides with the $\$$-module structure given by $\zeta \rightarrow-t_{\zeta^{*}}$. Since

$$
c_{\zeta *} \delta+\delta c_{\zeta *}=t_{\zeta *}
$$

it follows that the corresponding (5-module structure on the cohomology groups of our complexes is trivial. Hence, if the base field $F$ is of characteristic 0 and $G$ is irreducible, the induced action of $G$ on the cohomology groups of the complexes $A\left(T_{R}\right)$ and $A\left(T_{R^{K}}\right)$ is trivial.

By changing sides in the above definition of the $G$-module structure on $A\left(T_{R}\right)$, we obtain a second $G$-module structure; we indicate the operations of this structure by $\alpha \rightarrow x(\alpha)$, where, for $\alpha \epsilon A^{q}\left(T_{R}\right), x(\alpha)$ is defined by

$$
x(\alpha)\left(\tau_{1}, \cdots, \tau_{q}\right)=x \cdot \alpha\left(x^{-1} \cdot \tau_{1}, \cdots, x^{-1} \cdot \tau_{q}\right) .
$$

One sees immediately that this is the structure of a rational $G$-module, and that $\delta$ is a $G$-module endomorphism also for this new $G$-module structure. Let $A_{K}\left(T_{R}\right)$ denote the subset of all $\alpha \epsilon A\left(T_{R}\right)$ such that $x(\alpha)=\alpha$, for every $x \in K$, and $c_{\zeta}(\alpha)=0$, for every $\zeta \epsilon \Re$. The $(\xi$-module structure of $A\left(T_{R}\right)$ that is induced by our new $G$-module structure is given by $\zeta \rightarrow t_{\zeta}$, for every $\zeta \in \oiint^{\dagger}$. Hence, for each $\zeta \in \Re$, $t_{\zeta}$ annihilates $A_{K}\left(T_{R}\right)$, and the formula $\delta c_{\zeta}+c_{\zeta} \delta=t_{\zeta}$ shows that $A_{K}\left(T_{R}\right)$ is a subcomplex of $A\left(T_{R}\right)$. Evidently, it is also an $R^{K}$-submodule of $A\left(T_{R}\right)$. Finally, one checks easily that $A_{K}\left(T_{R}\right)$ is also a $G$-submodule of $A\left(T_{R}\right)$, for the $G$-module structure $\alpha \rightarrow x \cdot \alpha$ we defined originally.

We shall show that $\rho\left(A\left(T_{R^{K}}\right)\right) \subset A_{K}\left(T_{R}\right)$. It follows at once from the definitions that $x(\rho(\alpha))=\rho(\alpha)$, for every $\alpha \epsilon A\left(T_{R^{K}}\right)$ and every $x \in K$. There remains to show that $c_{\zeta} \circ \rho=0$, for all $\zeta \in \Omega$. Let $\alpha \epsilon A^{q}\left(T_{R^{K}}\right)$. Then we have

$$
\begin{aligned}
\left(c_{\zeta} \rho \alpha\right)\left(\tau_{2}, \cdots, \tau_{q}\right)(x) & =(\rho \alpha)\left(\zeta, \tau_{2}, \cdots, \tau_{q}\right)(x) \\
& =\alpha\left((x \cdot \zeta)^{*}, \beta\left(x \cdot \tau_{2}\right)^{*}, \cdots, \beta\left(x \cdot \tau_{q}\right)^{*}\right)(x) .
\end{aligned}
$$


Now the map $\tau \rightarrow \alpha\left(\tau, \beta\left(x \cdot \tau_{2}\right)^{*}, \cdots, \beta\left(x \cdot \tau_{q}\right)^{*}\right)$ is an element of $A^{1}\left(T_{R^{K}}\right)$. Hence we see that it suffices to prove that, for every $\alpha \in A^{1}\left(T_{R^{K}}\right)$ and every $x \epsilon G$, we have $\alpha\left((x \cdot \zeta)^{*}\right)(x)=0$. But

$$
\alpha\left((x \cdot \zeta)^{*}\right)(x)=\alpha\left(\zeta^{*} \cdot x^{-1}\right)(x)=\left(x^{-1} \cdot \alpha\right)\left(\zeta^{*}\right)(1) .
$$

Hence it suffices to show that $\alpha\left(\zeta^{*}\right)(1)=0$, for every $\alpha \in A^{1}\left(T_{R^{K}}\right)$.

Let $S$ denote the field of quotients of $R^{K}$, and choose a maximal set $\left(\zeta_{1}, \cdots, \zeta_{n}\right)$ of elements of $(S)$ such that the derivations of $S$ effected by $\zeta_{1}^{*}, \cdots, \zeta_{n}^{*}$ are linearly independent over $S$. We have $F \subset S \subset Q$, and $Q$ is finitely generated (as a field) over $F$. Hence $S$ is finitely generated over $F$. Hence there are elements $f_{1}, \cdots, f_{n}$ in $R^{K}$ such that the determinant formed from the $\zeta_{i}^{*}\left(f_{j}\right)$ is different from 0 . Let $D$ be this determinant. If we replace the $\zeta_{i}$ by $x^{-1} \cdot \zeta_{i}$, with $x \epsilon G$, and the $f_{j}$ by $f_{j} \cdot x, D$ is changed to $D \cdot x$. Hence we may choose the $\zeta_{i}$ and the $f_{j}$ so that $D(1) \neq 0$. Now if we consider the system of linear equations

$$
\sum_{j=1}^{n} s_{j} \zeta_{i}^{*}\left(f_{j}\right)=\alpha\left(\zeta_{i}^{*}\right),
$$

we find that there are elements $g_{j} \in R^{K}$ such that

$$
D \alpha\left(\zeta_{i}^{*}\right)=\sum_{j=1}^{n} g_{j} \zeta_{i}^{*}\left(f_{j}\right),
$$

for each $i$.

On the other hand, there is a nonzero element $g$ in $R^{K}$ such that $g \zeta^{*}$ coincides on $R^{K}$ with an $R^{K}$-linear combination of the $\zeta_{i}^{*}$. Hence

whence

$$
D \alpha\left(\zeta^{*}\right)=\sum_{j=1}^{n} g_{j} \zeta^{*}\left(f_{j}\right)
$$

$$
D(1) \alpha\left(\zeta^{*}\right)(1)=\sum_{j=1}^{n} g_{j}(1) \zeta^{*}\left(f_{j}\right)(1)=\sum_{j=1}^{n} g_{j}(1) \zeta\left(f_{j}\right)(1)=0,
$$

so that $\alpha\left(\zeta^{*}\right)(1)=0$, Q.E.D.

Proposition 3.1. Let $G$ be an irreducible algebraic linear group over the field $F$ of characteristic 0 , and let $K$ be a fully reducible algebraic subgroup of G. Then the map $\rho$ is an isomorphism of $A\left(T_{R} K\right)$ onto $A_{K}\left(T_{R}\right)$.

Proof. Regard $T_{R}$ as a $K$-module via the extended adjoint representation $\tau \rightarrow x \cdot \tau$. Let $I$ be the inverse image of $T_{R^{K}}$ for the restriction map of $T_{R}$ into the $R$-module of the $F$-derivations of $R^{K}$ into $R$. Clearly, $I$ is a $K$-submodule of $T_{R}$, and $\left(T_{R}\right)^{K} \subset I$. Now $T_{R}$, and hence $I$, are rational $K$-modules. Since $K$ is fully reducible, it follows that $I$ is semisimple as a $K$-module. With the trivial $K$-module structure on $T_{R^{K}}$, the restriction map $I \rightarrow T_{R^{K}}$ is evidently a $K$-module homomorphism. By Proposition 2.2, it is an epimorphism. Hence we conclude that the restriction map is an epimorphism of $\left(T_{R}\right)^{K}$ onto $T_{R^{K}}$. Now $\left(T_{R}\right)^{K}$ consists precisely of the $R^{K}$-linear combinations of the derivations $\zeta^{*}$, where $\zeta \in$ S. If $\alpha \epsilon A^{q}\left(T_{R^{R}}\right)$ and $\zeta_{1}, \cdots, \zeta_{q}$ are elements of $\$$, we have

$$
(\rho \alpha)\left(\zeta_{1}^{*}, \cdots, \zeta_{q}^{*}\right)=\alpha\left(\zeta_{1}^{*}, \cdots, \zeta_{q}^{*}\right) .
$$

Hence it is clear that $\rho$ is a monomorphism. 
Now let us recall from the proof of Lemma 2.2 that the kernel of the restriction map of $T_{R}$ into the $R$-module of the $F$-derivations of $R^{K}$ into $R$ is precisely $R \otimes \Omega$. It follows that, if $\gamma \in A_{K}^{q}\left(T_{R}\right)$ and $\tau_{1}, \cdots, \tau_{q}$ are elements of $T_{R}$, then $\gamma\left(\tau_{1}, \cdots, \tau_{q}\right)$ depends only on the restrictions of the $\tau_{i}$ 's to $R^{K}$. Moreover, since $x(\gamma)=\gamma$, for every $x \in K$, we have $\gamma\left(\tau_{1}, \cdots, \tau_{q}\right) \epsilon R^{K}$, whenever the $\tau_{i}$ 's belong to $\left(T_{R}\right)^{K}$. Hence the restriction of $\gamma$ to $q$-tuples of elements of $\left(T_{R}\right)^{K}$ induces an element $\alpha \epsilon A^{q}\left(T_{R^{K}}\right)$. We have

$$
(\rho \alpha)\left(\zeta_{1}^{*}, \cdots, \zeta_{q}^{*}\right)=\gamma\left(\zeta_{1}^{*}, \cdots, \zeta_{q}^{*}\right),
$$

whenever the $\zeta_{i}$ belong to (\$). It follows that $\rho(\alpha)=\gamma$, and we have shown that $\rho$ is an epimorphism. This completes the proof of Proposition 3.1.

Let $V$ be a rational $G$-module, and consider the rational $G$-module complex $A\left(T_{R}\right) \otimes V$. If $\gamma$ is an element of $A^{q}\left(T_{R}\right)$ and $v$ is an element of $V$, then $\gamma \otimes v$ defines an element of the space $C^{q}(\mathcal{S}, V)$ of the alternating $q$-cochains for (s) in $V$ by

$$
(\gamma \otimes v)\left(\zeta_{1}, \cdots, \zeta_{g}\right)=\gamma\left(\zeta_{1}, \cdots, \zeta_{q}\right)(1) v .
$$

Thus we have a map $\psi$ of $A\left(T_{R}\right) \otimes V$ into $C(\$, V)$. It has been shown in [5, Section 5] (and is actually easy to verify directly) that the restriction of $\psi$ to $\left(A\left(T_{R}\right) \otimes V\right)^{G}$ is an isomorphism of the complex $\left(A\left(T_{R}\right) \otimes V\right)^{G}$ onto the complex $C(\$, V)$.

Now we consider the $K$-module structure of $\left(A\left(T_{R}\right) \otimes V\right)^{G}$ obtained by using the operations $\alpha \rightarrow x(\alpha)$ on $A\left(T_{R}\right)$ and the trivial action on $V$. On $\left.C^{q}(\$) V\right)$, we introduce the usual $K$-module structure given by

$$
(x \cdot \varphi)\left(\zeta_{1}, \cdots, \zeta_{q}\right)=x \cdot \varphi\left(x^{-1} \cdot \zeta_{1}, \cdots, x^{-1} \cdot \zeta_{q}\right) .
$$

We claim that, for these $K$-module structures, the restriction of $\psi$ to $\left(A\left(T_{R}\right) \otimes V\right)^{G}$ is a $K$-module isomorphism. Let $x \epsilon K$, and let $\gamma$ and $v$ be as above. Then we have

$$
\begin{aligned}
\psi(x(\gamma \otimes v))\left(\zeta_{1}, \cdots, \zeta_{q}\right) & =x(\gamma)\left(\zeta_{1}, \cdots, \zeta_{q}\right)(1) v \\
& =\gamma\left(x^{-1} \cdot \zeta_{1}, \cdots, x^{-1} \cdot \zeta_{q}\right)(x) v \\
& =\left(x^{-1} \cdot \gamma\right)\left(x^{-1} \cdot \zeta_{1}, \cdots, x^{-1} \cdot \zeta_{q}\right)(1) v
\end{aligned}
$$

Hence, if $\alpha \epsilon\left(A^{q}\left(T_{R}\right) \otimes V\right)^{q}$, this gives

$$
\begin{aligned}
\psi(x(\alpha))\left(\zeta_{1}, \cdots, \zeta_{q}\right) & =x \cdot\left(\alpha\left(x^{-1} \cdot \zeta_{1}, \cdots, x^{-1} \cdot \zeta_{q}\right)(1) v\right) \\
& =(x \cdot \psi(\alpha))\left(\zeta_{1}, \cdots, \zeta_{q}\right)
\end{aligned}
$$

Thus our isomorphism of complexes $\psi:\left(A\left(T_{R}\right) \otimes V\right)^{G} \rightarrow C(\$, V)$ is also a $K$-module isomorphism. Hence we see immediately that $\psi$ maps $\left(A_{K}\left(T_{R}\right) \otimes V\right)^{G}$ isomorphically onto $C(\$ / \Omega, V)^{K}$. Under the conditions of Proposition 3.1, we compose $\psi$ with the isomorphism of $\left(A\left(T_{R} K\right) \otimes V\right)^{G}$ onto $\left(A_{K}\left(T_{R}\right) \otimes V\right)^{G}$ that is induced by the isomorphism $\rho$ of Proposition 
3.1 to obtain an isomorphism of the complex $\left(A\left(T_{R^{K}}\right) \otimes V\right)^{G}$ onto the complex $C(\$ / \Omega, V)^{K}$.

Let $K_{1}$ be the irreducible component of the identity in $K$. Then $C(\$ / \Omega, V)^{K}$ is the $K / K_{1}$-fixed part of $C(\$ / \Omega, V)^{K_{1}}$, which is the $\Omega$-annihilated part of $C(\$ / \Omega, V)$. The cohomology space of the complex $C(\$ / \Omega, V)^{K_{1}}$ is the relative Lie algebra cohomology space $H(\$, \Omega, V)$ for $(\$, \Omega)$ in $V$. As a module for the finite group $K / K_{1}, C(\$ / \Re, V)^{K_{1}}$ is semisimple. Hence the $K / K_{1}$-fixed part of $H(S, \Omega, V)$ is the cohomology space of the complex $C(\$ / \Omega, V)^{K}$. Hence we have the following result.

Theorem 3.1. Let $G$ be an irreducible algebraic linear group over the field $F$ of characteristic 0 , and let $K$ be a fully reducible algebraic subgroup of $G$. Then, for any rational G-module $V$, the cohomology space of the complex $\left(A\left(T_{R^{K}}\right) \otimes V\right)^{G}$ is isomorphic, via the maps $\rho$ and $\psi$ defined above, with the $K$-fixed part $H(\$, \Omega, V)^{K}$ of the relative Lie algebra cohomology space for (\$, $\Omega$ ) in $V$.

In the special case where $G$ is fully reducible, Theorem 3.1 gives a determination of the cohomology space of the complex $A\left(T_{R^{K}}\right)$. In that case, the rational $G$-module $A\left(T_{R} K\right)$ is semisimple. Since the action of $G$ on the cohomology space of the complex $A\left(T_{R^{K}}\right)$ is trivial, this implies that the injection $A\left(T_{R^{K}}\right)^{G} \rightarrow A\left(T_{R^{K}}\right)$ induces an isomorphism of the cohomology spaces. Hence, if we take for $V$ the trivial $G$-module $F$, Theorem 3.1 gives the following result.

Theorem 3.2. Let $G$ be an irreducible algebraic linear group over the field $F$ of characteristic 0 . Suppose that $G$ is fully reducible, and let $K$ be a fully reducible algebraic subgroup of $G$. Then the cohomology space of the complex $A\left(T_{R^{K}}\right)$ is isomorphic with $H((), \Re, F)^{K}$.

In the general case of Theorem 3.1, there is a spectral sequence linking the tensor product of the cohomology space of the differential forms on $R^{K}$ and the space of the rational cohomology for $G$ in $V$ to the relative Lie algebra cohomology space for ( $(S, \Omega)$ in $V$. In order to derive this result, we need more information on the $G$-module $A\left(T_{R^{K}}\right)$.

Under the present assumptions, $A\left(T_{R} K\right)$ may be identified with $A_{K}\left(T_{R}\right)$, and we shall show that $A_{K}\left(T_{R}\right)$ is a direct $G$-module summand of $A\left(T_{R}\right)$. Since $K$ is fully reducible, we have a direct $K$-module decomposition (S) $=\Re+\mathfrak{B}$. For each $q>0$, the $R$-module $A^{q}\left(T_{R}\right)$ is the direct sum of two $R$-submodules $A_{\Re}^{q}\left(T_{R}\right)$ and $A_{\mathfrak{P}}^{q}\left(T_{R}\right)$, consisting of the elements annihilated by the $c_{\zeta}$ with $\zeta \in \Re$, or of the elements annihilated by the $c_{\zeta}$ with $\zeta \in \mathfrak{B}$, respectively. Since $\Re$ and $\mathfrak{B}$ are $K$-submodules of $(\mathfrak{S}$, it is clear that these two $R$-submodules are stable under the operations $\alpha \rightarrow x(\alpha)$, with $x \in K$. With reference to this $K$-action, the $K$-fixed part of $A^{q}\left(T_{R}\right)$ is therefore the direct sum of $A_{K}^{q}\left(T_{R}\right)$ and the $K$-fixed part of $A_{\mathfrak{B}}^{q}\left(T_{R}\right)$, and it is clear that 
this is a direct $G$-module decomposition for the $G$-module structure $\alpha \rightarrow x \cdot \alpha$. Hence $A_{K}\left(T_{R}\right)$ is a direct $G$-module summand of the $K$-fixed part (for the action $\alpha \rightarrow x(\alpha)$ ) of $A\left(T_{R}\right)$. Since $K$ is fully reducible, the rational $K$-module $A\left(T_{R}\right)$ (for the action $\left.\alpha \rightarrow x(\alpha)\right)$ is semisimple. Hence the $K$-fixed part of $A\left(T_{R}\right)$ is a direct $G$-module summand of $A\left(T_{R}\right)$. Thus $A_{K}\left(T_{R}\right)$ is indeed a direct $G$-module summand of $A\left(T_{R}\right)$.

It follows at once from [5, Proposition 2.2] and from the form in which $A\left(T_{R}\right)$ is exhibited in [5] that, for every rational $G$-module $V$, the $G$-module $A\left(T_{R}\right) \otimes V$ is rationally injective, in the sense of [5]. In virtue of what we have just proved, this implies that, under the assumptions of Theorem 3.1, the G-module $A\left(T_{R^{K}}\right) \otimes V$ is rationally injective.

Put $U^{q}=A^{q}\left(T_{R^{K}}\right) \otimes V, U=\sum_{q} U^{q}$. Then $U$ is a rational $G$-module, and we consider the complex $C(G, U)=\sum_{p} C^{p}(G, U)$ of the nonhomogeneous rational representative cochains for $G$ in $U$, in the sense of [5, Section $2]$. Let $\delta_{G}$ denote the coboundary operator of this complex, and let $\delta_{U}$ denote the coboundary operator of the complex $U$. Then $C(G, U)$ has the structure of a double complex of rational $G$-modules, with total coboundary operator $\delta=\delta_{G}+(-1)^{p} \delta_{U}$, on $C^{p}(G, U)$.

Since $U$ is rationally injective as a $G$-module, the rational cohomology groups $H^{n}(G, U)$ for $G$ in $U$ are $(0)$, for all $n>0$. A standard argument [3, Proposition 4], shows that the injection $U^{G} \rightarrow C^{0}(G, U) \subset C(G, U)$ induces an isomorphism of $H^{n}\left(U^{G}, \delta_{U}\right)$ onto $H^{n}(C(G, U), \delta)$, for all $n \geqq 0$, where the grading on $C(G, U)$ is given by $C(G, U)^{n}=\sum_{p+q=n} C^{p}\left(G, U^{q}\right)$.

We introduce a decreasing filtration $\left(L_{i}\right)$ on this double complex, where $L_{i}=\sum_{p \geqq i} C^{p}(G, U)$. The spectral sequence derived from this filtration is the spectral sequence of Cartan-Leray, with the ordinary group cohomology replaced by the rational cohomology of $G$. The arguments and results of [3, Chapter I, Section 5] apply without change to the present situation, giving the following result. Let $H\left(U^{G}\right)_{p}$ denote the subspace of $H\left(U^{G}\right)$ whose image in $H(C(G, U))$ is the image of $H\left(L_{p}\right)$. Then the limit $E_{\infty}$ of the spectral sequence is given by

$$
E_{\infty}^{p, q}=H^{p+q}\left(U^{G}\right)_{p} / H^{p+q}\left(U^{G}\right)_{p+1} ;
$$

the term $E_{2}$ of the spectral sequence is given by

$$
E_{2}^{p, q}=H^{p}\left(G, H^{q}(U)\right) .
$$

Now we have $H^{q}(U)=H^{q}\left(A\left(T_{R^{K}}\right)\right) \otimes V$, and we have seen earlier that the action of $G$ on $H^{q}\left(A\left(T_{R^{K}}\right)\right)$ is trivial. Hence we obtain

$$
E_{2}^{p, q}=H^{p}(G, V) \otimes H^{q}\left(A\left(T_{R} K\right)\right) .
$$

On the other hand, by Theorem $\left.3.1, H\left(U^{G}\right)=H(\$), ~ \Re, V\right)^{K}$.

Thus we have the following result, which is the analogue of a result for Lie groups due to van Est [7, Theorem 3]. 
Theorem 3.3. Let $G$ be an irreducible algebraic linear group over a field $F$ of characteristic 0 , and let $K$ be a fully reducible algebraic subgroup of $G$. Let $V$ be a rational G-module. Then there is a spectral sequence with

$$
E_{2}=H(G, V) \otimes H\left(A\left(T_{R}\right)\right)
$$

and with $E_{\infty}$ the graded space obtained from a filtration of $H(\$, \Omega, V)^{K}$.

\section{Relative Lie algebra cohomology}

While the connection expressed in Theorem 3.3 is rather vague, we have very precise connections of this type in two special cases. One of these is the case where $G$ is fully reducible, which case is covered by Theorem 3.2. The other is the case where $K$ is a maximal fully reducible subgroup of $G$. In this case, the spectral sequence of Theorem 3.3 collapses, in the sense that $E_{2}$ coincides with $E_{\infty}, H\left(A\left(T_{R^{K}}\right)\right)=H^{0}\left(A\left(T_{R^{K}}\right)\right)=F$, and the result becomes a superficially different form of the isomorphism of [5, Theorem 5.2]. In order to express the relative Lie algebra cohomology in terms of the cohomology of differential forms on an algebra of representative functions and the rational cohomology of $G$, we make a reduction to the above special cases on the level of the relative Lie algebra cohomology. The reduction is based on an imbedding of the given fully reducible subgroup of $G$ in a maximal fully reducible subgroup of $G$, which enables us to use an easy generalization of the tensor product decomposition of the Lie algebra cohomology given by [4, Theorem 13].

TheOREM 4.1. Let (\$) be a finite-dimensional Lie algebra over the field $F$ of

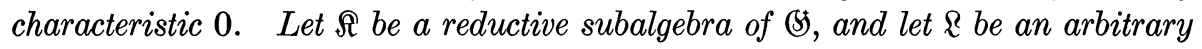
subalgebra of $\Omega$. Suppose that the restriction map $H((\$), R, F) \rightarrow H(\Omega, \mathfrak{R}, F)$ is an epimorphism. Let $V$ be a finite-dimensional (\$-module that is semisimple as a $\Re$-module. Then, for each $n \geqq 0, H^{n}(\$, \&, V)$ is isomorphic with

$$
\sum_{p+q=n} H^{p}(\Re, \Re, F) \otimes H^{q}(\$, \Re, V) ;
$$

an isomorphism of the second space onto the first is obtained in the natural way from any homogeneous linear monomorphism $H(\Omega, R, F) \rightarrow H((S, R, F)$ inverse to the restriction epimorphism, the canonical map $H($ (S, $\Re, V) \rightarrow H(\mathbb{S}, \mathbb{R}, V)$,

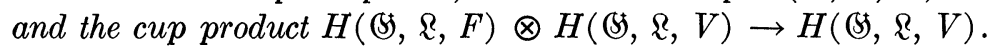

The proof is almost identical with the proof of [4, Theorem 12]. It requires the following generalization of [4, Theorem 10].

Lemma 4.1. Let $\Omega$ be a finite-dimensional reductive Lie algebra over the field $F$ of characteristic 0 , and let $M$ be a finite-dimensional semisimple $\Re$-module such that $M^{\Re}=(0)$. Then, if $\Omega$ is any subalgebra of $\Re, H^{n}(\Re, \Re, M)=(0)$, for all $n \geqq 0$.

Proof. We may evidently suppose that $M$ is simple and that $n>0$. Let $\mathfrak{C}$ be the center of $\Omega$. Then $\Omega$ is the direct sum of [ $\Omega, \Omega]$ and $\mathfrak{C}$, and 
$[\Omega, \Omega]$ is semisimple. For every $\gamma \in \mathbb{S}, \gamma \cdot M$ is a $\Re$-submodule of $M$, and thus is either $(0)$ or $M$. Suppose first that $\gamma \cdot M=M$. Let $f$ be a relative $n$ cocycle for $(\Omega, \Omega)$ in $M$. Then $c_{\gamma}(f)$ is a relative $(n-1)$-cochain for $(\Omega, \Omega)$ in $M$, and $\delta\left(c_{\gamma}(f)\right)=\gamma \cdot f$. But $\gamma$ acts as a (5)-module automorphism $\rho_{\gamma}$ on $M$, and $\gamma \cdot f=\rho_{\gamma} \circ f$. Hence we have $f=\rho_{\gamma}^{-1} \circ \delta\left(c_{\gamma}(f)\right)=\delta\left(\rho_{\gamma}^{-1} \circ c_{\gamma}(f)\right)$, and $\rho_{\gamma}^{-1} \circ c_{\gamma}(f)$ is evidently a relative $(n-1)$-cochain for $(\Re, \mathfrak{l})$ in $M$. Hence we may now suppose that $\mathfrak{S} \cdot M=(0)$.

Let $\mathfrak{T}$ denote the annihilator of $M$ in $\Omega$. Then $\mathfrak{T}$ contains $\mathfrak{S}$, whence $\Omega$ is a direct sum $\mathfrak{T}+\mathfrak{S}$, where $\mathfrak{\Im}$ is a semisimple ideal of $\Re$ and the representation of $\Im$ on $M$ is faithful. Let $\psi$ be the Casimir operator of this representation. If $\zeta \rightarrow \rho_{\zeta}$ is the representation of $\Omega$ on $M$, we have $\psi=\sum_{i} \rho_{\zeta_{i}} \circ \rho_{\tau_{i}}$, where $\zeta_{i}$ and $\tau_{i}$ are elements of $S$ and $\sum_{i}\left(\left[\alpha, \zeta_{i}\right] \otimes \tau_{i}+\zeta_{i} \otimes\left[\alpha, \tau_{i}\right]\right)=0$, for all $\alpha \epsilon \Re$. Now put $g=\sum \rho_{\zeta_{i}} \circ c_{\tau_{i}}(f)$. Then one shows by a familiar computation (see [2, p. 118]) that $\delta(g)=\psi \circ f$, so that

$$
f=\psi^{-1} \circ \delta(g)=\delta\left(\psi^{-1} \circ g\right) \text {. }
$$

Hence there remains only to see that $\psi^{-1} \circ g$ is a relative cochain for $(\Re, \mathfrak{R})$ in $M$. Clearly, $c_{\zeta}\left(\psi^{-1} \circ g\right)=0$, for every $\zeta \in \mathbb{R}$. Also

$$
\delta\left(c_{\zeta}\left(\psi^{-1} \circ g\right)\right)=\zeta \cdot\left(\psi^{-1} \circ g\right)-c_{\zeta}\left(\delta\left(\psi^{-1} \circ g\right)\right)=\zeta \cdot\left(\psi^{-1} \circ g\right)-c_{\zeta}(f) .
$$

Taking $\zeta \in \Re$, we see from this that $\zeta \cdot\left(\psi^{-1} \circ g\right)=0$. Thus $\psi^{-1} \circ g$ is indeed a relative cochain, and the proof of Lemma 4.1 is complete.

In order to prove Theorem 4.1, one can now proceed in exactly the same way as in [4], replacing the ordinary cochain complex $C(S, V)$ by the relative complex $C(\$), \mathfrak{R}, V)=C(\$) / R, V)^{\mathfrak{R}}$. One considers the filtration of this complex that is obtained by intersecting the filtration groups used in [4] with $C(B, \Omega, V)$. For the corresponding spectral sequence, one shows first that $\left.E_{1}^{p, q}=H^{q}\left(\Re, \Omega, C^{p}(\$) / \Omega, V\right)\right)$. Here, the only deviation from the proof in [4] is that the projection $\gamma \rightarrow \gamma^{*}$ of $\$ 5$ onto $\Re$ used in the proof of $[4$, Theorem 1] must now be chosen so that it is a $\Re$-module projection, which is possible, because $\Omega$ is reductive in (S). Next one proceeds to show, as in the proof of [4, Theorem 11 and Corollary], that $E_{2}^{p, q}=H^{q}(\Re, \Re, F) \otimes H^{p}(\$, \Omega, V)$, replacing the use of [4, Theorem 10] with an appeal to Lemma 4.1 above. The rest of the proof of Theorem 4.1 is exactly as the proof of [4, Theorem 12], where one now ignores the mutiplicative feature of the isomorphism to be established.

Now suppose that there is an ideal $\mathfrak{N}$ in $(\$)$ such that $\$(S$ is the semidirect sum $\Re+\Re$, where $\Re$ is a reductive subalgebra of $(5)$. Let $\mathfrak{R}$ be any subalgebra of $\Omega$. Then it is easy to see that the restriction map

$$
H((S), R, F) \rightarrow H(\Re, \Re, F)
$$

is an epimorphism. Furthermore, it is seen exactly as in [4, p. 603] that $H(\$, \Re, V)$ is naturally isomorphic with $H(\Re, V)^{\mathscr{S}}$, for every (\$)-module $V$ 
that is semisimple as a $\Omega$-module. Hence Theorem 4.1 shows that $H(\$, \&, V)$ is isomorphic with $H(\Re, \Re, F) \otimes H(\Re, V)^{\mathfrak{B}}$.

Now let $G$ be an irreducible algebraic linear group over the field $F$ of characteristic 0 . Let $V$ be a finite-dimensional rational $G$-module, and let $L$ be a fully reducible algebraic subgroup of $G$. Let $K$ be a maximal fully reducible subgroup of $G$ that contains $L$. Then $K$ is irreducible as an algebraic linear group, and $G$ is the semidirect product $K \cdot N$, where $N$ is the maximum unipotent normal subgroup of $G$. Now if $(5, \Re, \Omega, \Re$ are the Lie algebras of $G, K, L, N$, respectively, all of our above assumptions hold. The algebra of the rational representative functions on $K$ may be identified with $R^{N}$. Hence Theorem 3.2 shows that $H(\Omega, \&, F)^{L}$ is isomorphic with $H\left(A\left(T_{R} L \cdot N\right)\right)$. On the other hand, by [5, Theorem 5.2], the cohomology space $H(\mathfrak{N}, V)^{\mathfrak{G}}$ is isomorphic with the space $H(G, V)$ of the rational cohomology for $G$ in $V$. Passing to the $L$-fixed parts in the above isomorphism result for the Lie algebra cohomology, we obtain the result that $H(\$, \mathbb{R}, V)^{L}$ is isomorphic with $H(G, V) \otimes H\left(A\left(T_{R} L \cdot N\right)\right)$. Thus the change from the algebra $R^{L}$ to the subalgebra $R^{L \cdot N}$ closes the gap left by Theorem 3.3, and we have the following result.

Theorem 4.2. Let $G$ be an irreducible algebraic linear group over a field $F$ of characteristic 0 , and let $K$ be a fully reducible algebraic subgroup of $G$. Let $V$ be a finite-dimensional rational $G$-module, and let $N$ be the maximum unipotent normal subgroup of $G$. Then $H(\xi), \Re, V)^{K}$ is isomorphic with $H(G, V) \otimes H\left(A\left(T_{R} K \cdot N\right)\right)$.

\section{The universal differential forms}

Let $P$ be a commutative unitary $F$-algebra, where $F$ is a field. Besides the complex $A\left(T_{P}\right)$ which we defined at the beginning of Section 3 , one can define a formally similar complex without referring to the derivations of $P$. This construction, which is well known, is as follows. $\operatorname{Regard} P \otimes P$ as a $P$-module such that $a(b \otimes c)=(a b) \otimes c$. Let $J$ be the submodule that is generated over $P$ by the elements of the form $1 \otimes(a b)-a \otimes b-b \otimes a$. Let $D_{P}^{1}$ denote the factor module $(P \otimes P) / J$. The elements of $D_{P}^{1}$ are called the universal differential forms of degree 1 on $P$. It is immediately verified that the $P$-module $T_{P}$ of all $F$-derivations of $P$ is naturally isomorphic with $\operatorname{Hom}_{P}\left(D_{P}^{1}, P\right)$; the isomorphism is induced by attaching to each $\tau \in T_{P}$ the map $P \otimes P \rightarrow P$ that sends $a \otimes b$ onto $a \tau(b)$.

We define an $F$-derivation $d: P \rightarrow D_{P}^{1}$ by $d(a)=1 \otimes a+J_{.}$Then $d(P)$ evidently generates $D_{P}^{1}$ over $P$. Put $D_{P}^{0}=P$, and let $D_{P}=\sum_{q} D_{P}^{q}$ denote the exterior $P$-algebra built over $D_{P}^{1}$. It is easily verified that the map $d: D_{P}^{0} \rightarrow D_{P}^{1}$ has a unique extension, still denoted $d$, to a homogeneous $F$-linear antiderivation of degree 1 and square 0 on $D_{P}$. The complex $\left(D_{P}, d\right)$ is called the complex of the universal differential forms on $P$.

The dual of the natural $P$-module isomorphism $T_{P} \rightarrow \operatorname{Hom}_{P}\left(D_{P}^{1}, P\right)$, 
preceded by the canonical $P$-module homomorphism of $D_{P}^{1}$ into its bidual, gives a natural $P$-module homomorphism $\varphi: D_{P}^{1} \rightarrow A^{1}\left(T_{P}\right)$. For $f \in P$ and $\tau \epsilon T_{P}$, we have $\varphi(d f)(\tau)=\tau(f)$. The map $\varphi$ extends canonically to a homogeneous $P$-module homomorphism of $D_{P}$ into $A\left(T_{P}\right)$, still denoted $\varphi$, such that $\varphi \circ d=\delta \circ \varphi$, i.e., $\varphi$ extends to a homomorphism of the complex $\left(D_{p}, d\right)$ into the complex $\left(A\left(T_{P}\right), \delta\right)$. It can be shown in an elementary fashion that, if $D_{P}^{1}$ is finitely generated and projective as a P-module, then $\varphi$ is an isomorphism. We shall see that this is the case for $P=R^{K}$, where $R$ is the algebra of the rational representative functions on the irreducible algebraic linear group $G$, and $K$ is a fully reducible algebraic subgroup of $G$, the base field $F$ being of characteristic 0 .

Lemma 5.1. Let $G$ be an irreducible algebraic linear group over the field $F$ of characteristic 0 , and let $K$ be a fully reducible algebraic subgroup of $G$. Then $R^{K}$ is finitely generated as an F-algebra.

Proof. Let $G$ be given as an algebraic group of linear automorphisms of determinant 1 of a finite-dimensional $F$-space $V$. Let $P$ be the algebra of all polynomial functions on the space of all linear endomorphisms of $V$, and let $\rho$ denote the restriction epimorphism $P \rightarrow R$. Let $I$ be the kernel of $\rho$. We regard $P$ as a $K$-module, with $K$ operating by left translation. Then, since $K$ is fully reducible, $P$ is semisimple as a $K$-module. By the fundamental theorem of invariants, $P^{K}$ is therefore finitely generated as an $F$-algebra. Since $P$ is semisimple as a $K$-module, $\rho$ induces an epimorphism of $P^{K}$ onto $R^{K}$, whence $R^{K}$ is finitely generated.

Lemma 5.2. In addition to the assumptions of Lemma 5.1, assume that $F$ is algebraically closed. Then every homomorphism $R^{K} \rightarrow F$ leaving the constants fixed is of the form $f \rightarrow f(x)$, with some $x \in G$.

Proof. Let $\varphi$ be such a homomorphism, and let $I$ be the kernel of $\varphi$. We have a direct $K$-module decomposition $R=R^{K}+S$, where $S$ consists of the elements of the form $\sum(x \cdot f-f)$, with $f \in R$ and $x \in K$. This shows that $R^{K} S=S$. Now we have $I R=I R^{K}+I S \subset I+S$. If we had $1 \epsilon I R$, we could accordingly write $1=i+s$, with $i \epsilon I$ and $s \in S$. But then $s=$ $1-i \epsilon R^{K}$, whence $s=0$ and $1 \epsilon I$; a contradiction. Thus we have $I R \neq R$. Let $J$ be a maximal ideal of $R$ containing $I R$. Then, since $I$ is a maximal ideal of $R^{K}$, we have $I=J \cap R^{K}$. Since $R$ is finitely generated and $F$ is algebraically closed, we have $R / J=F$. Hence we conclude that $\varphi$ extends to a homomorphism $R \rightarrow F$. But this is of the form $f \rightarrow f(x)$, with some $x \epsilon G$, so that Lemma 5.2 is proved.

Combining Lemma 5.1 with Lemma 5.2, we have immediately the following result.

THeorem 5.1. Let $G$ be an irreducible algebraic linear group over an algebraically closed field of characteristic 0 . Let $K$ be a fully reducible algebraic 
subgroup of $G$. Then the set $G / K$ of the cosets $x K$ has the structure of an affine algebraic variety, with $R^{K}$ as the algebra of the polynomial functions.

Lemma 5.3. Let $G$ be an irreducible algebraic linear group over the field $F$ of characteristic 0 , and let $K$ be a fully reducible algebraic subgroup of $G$. Then the canonical $\operatorname{map} \varphi: D_{R}^{1} \mathrm{~K} \rightarrow A^{1}\left(T_{R^{K}}\right)$ is an isomorphism.

Proof. By extending the base field $F$ to its algebraic closure and using the remarks made at the end of our proof of Proposition 2.2, we see that no generality is lost in assuming $F$ to be algebraically closed, which we shall now do.

Let $\alpha \in D_{R}^{1} K$, and suppose that $\varphi(\alpha)=0$. We may write

$$
\alpha=\sum_{i=1}^{q} a_{i} d\left(b_{i}\right) \text {, }
$$

where $a_{i}$ and $b_{i}$ are elements of $R^{K}$, and the $b_{i}$ are different from 0 . We can choose elements $f_{1}, \cdots, f_{n}$ in $R^{K}$ so that they are algebraically independent over $F$ and $R^{K}$ is algebraic over $F\left[f_{1}, \cdots, f_{n}\right]$. Then there are $F$-derivations $\sigma_{1}, \cdots, \sigma_{n}$ of the field of quotients $Q^{K}$ of $R^{K}$ such that $\sigma_{i}\left(f_{j}\right)=\delta_{i j}$. Since $R^{K}$ is finitely generated over $F$, we can find a nonzero element $f \in R^{K}$ such that each $f \sigma_{i}$ sends $R^{K}$ into itself. Thus we obtain elements $\tau_{1}, \cdots, \tau_{n}$ of $T_{R} K$ such that the determinant formed with the elements $\tau_{i}\left(f_{j}\right)$ is different from 0 .

Now let $p_{i}$ be a nonzero polynomial with coefficients in $F\left[f_{1}, \cdots, f_{n}\right]$ and of minimal degree such that $p_{i}\left(b_{i}\right)=0$. Let $p_{i j}$ denote the polynomial obtained from $p_{i}$ by differentiating its coefficients with respect to $f_{j}$, and let $p_{i}^{\prime}$ denote the ordinary derivative of the polynomial $p_{i}$. Then we have

$$
p_{i}^{\prime}\left(b_{i}\right) d\left(b_{i}\right)+\sum_{j=1}^{n} p_{i j}\left(b_{i}\right) d\left(f_{j}\right)=0, \text { and } p_{i}^{\prime}\left(b_{i}\right) \neq 0 .
$$

Put $p=p_{1}^{\prime}\left(b_{1}\right) \cdots p_{q}^{\prime}\left(b_{q}\right)$. Then $p \neq 0$, and we have

$$
p \alpha=\sum_{i=1}^{n} g_{i} d\left(f_{i}\right), \quad \text { with } g_{i} \in R^{K} \text {. }
$$

Since $\varphi(\alpha)=0$, we have

$$
0=p \alpha\left(\tau_{k}\right)=\sum_{i=1}^{n} g_{i} \tau_{k}\left(f_{i}\right), \quad \text { for each } k .
$$

Since the determinant formed from the coefficients of the $g_{i}$ here is not 0 , this gives $g_{i}=0$, for each $i$, whence $p \alpha=0$.

Make $R^{K} \otimes R^{K}$ into a $G$-module such that $x \cdot(f \otimes g)=\left(f \cdot x^{-1}\right) \otimes\left(g \cdot x^{-1}\right)$. Then the kernel of the canonical epimorphism of $R^{K} \otimes R^{K}$ onto $D_{R K}^{1}$ is evidently $G$-stable, so that we get an induced $G$-module structure on $D_{R}^{1}$. Clearly, the $F$-space spanned by the transforms of any element of $D_{R}^{1}$ is finite-dimensional. Applying the above argument to a finite number of transforms of $\alpha$, we see therefore that there is a nonzero element $f$ in $R^{K}$ such that $f(x \cdot \alpha)=0$, for every $x \epsilon G$. Hence $(f \cdot x) \alpha=0$, for every $x \epsilon G$.

Now let $I$ be the ideal of all $g \in R^{K}$ such that $g \alpha=0$. Our last result shows that $I$ has no zero on $G$. By Theorem 5.1, this means that $I=R^{K}$, whence $\alpha=0$. Thus we have shown that $\varphi$ is a monomorphism. 
Finally, we observe that the argument that just precedes the statement of Proposition 3.1 shows that, if $\gamma$ is any given element of $A^{1}\left(T_{R} R\right)$, the ideal of all $g \in R^{K}$ such that $g \gamma \epsilon \varphi\left(D_{R K}^{1}\right)$ has no zero on $G$, whence we conclude that $\varphi$ is an epimorphism. This completes the proof of Lemma 5.3.

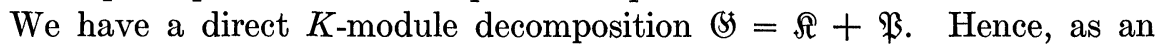
$R^{K}$-module, $\left(R \otimes(5)^{K}\right.$ is the direct sum of $(R \otimes \Re)^{K}$ and $(R \otimes \mathfrak{B})^{K}$. Now $T_{R^{K}}$ is isomorphic, as an $R^{K}$-module, with $\left(R \otimes(\$ / \Re)^{K}\right.$, and hence with $(R \otimes \mathfrak{P})^{K}$. Thus we see that $T_{R^{K}}$ is isomorphic with a direct $R^{K}$-module summand of $(R \otimes(5))^{K}$. On the other hand, $(R \otimes(5))^{K}$ may be identified with $R^{K} \otimes\left(\mathcal{S}^{*}\right.$. Hence $T_{R^{K}}$ is a direct $R^{K}$-module summand of a finitely generated free $R^{K}$-module. In other words, $T_{R^{K}}$ is a finitely generated projective $R^{K}$-module.

If $M$ is any $R^{K}$-module, let $E(M)$ denote the exterior $R^{K}$-algebra built over $M$. Evidently, $A\left(T_{R^{K}}\right)$ may be identified with $\operatorname{Hom}_{R^{K}}\left(E\left(T_{R^{K}}\right), R^{K}\right)$. On the other hand, if $M$ is a free $R^{K}$-module with a finite $R^{K}$-basis, the canonical map $E\left(\operatorname{Hom}_{R^{K}}\left(M, R^{K}\right)\right) \rightarrow \operatorname{Hom}_{R^{K}}\left(E(M), R^{K}\right)$ is an isomorphism. Since $T_{R K}$ is a direct $R^{K}$-module summand of such an $M$, it follows that the canonical map $E\left(A^{1}\left(T_{R^{K}}\right)\right) \rightarrow \operatorname{Hom}_{R^{K}}\left(E\left(T_{R^{K}}\right), R^{K}\right)$ is also an isomorphism. Thus, using the above identification, the canonical map of $E\left(A^{1}\left(T_{R^{K}}\right)\right)$ into $A\left(T_{R^{K}}\right)$ is an isomorphism. Taking account of Lemma 5.3, we have the following result.

THeorem 5.2. Let $G$ be an irreducible algebraic linear group over a field of characteristic 0 , and let $K$ be a fully reducible algebraic subgroup of $G$. Then the canonical map $\varphi$ of the complex $D_{R^{K}}$ of the universal differential forms on $R^{K}$ into the complex $A\left(T_{R} K\right)$ of the differential forms based on the F-derivations of $R^{K}$ is an ismorphism. Moreover, these complexes are finitely generated projective $R^{K}$-modules, and the homogeneous components of degree larger than the dimension of $(\$ / \Re$ are $(0)$.

Only the last statement of this theorem still requires verification. Let $q$ be the dimension of $\$(\Omega)$. Then we can find elements $f_{1}, \cdots, f_{q}$ in $R^{K}$ such that $R^{K}$ is algebraic over $F\left[f_{1}, \cdots, f_{q}\right]$. Let $g_{1}, \cdots, g_{q+1}$ be arbitrary elements of $R^{K}$. By an argument we used in proving Lemma 5.3, we see that there is a nonzero element $p_{i}$ in $R^{K}$ such that $p_{i} d\left(g_{i}\right)$ can be written in the form $\sum_{j=1}^{q} a_{j} d\left(f_{j}\right)$, with $a_{j} \in R^{K}$. Hence we find that

$$
p_{1} \cdots p_{q+1} d\left(g_{1}\right) \cdots d\left(g_{q+1}\right)=0 .
$$

Since $D_{R K}^{q+1}$ is a projective $R^{K}$-module, this implies that $d\left(g_{1}\right) \cdots d\left(g_{q+1}\right)=0$, Q.E.D.

\section{REFERENCES}

1. C. Chevalley, Théorie des groupes de Lie, II, Paris, Hermann, 1951.

2. C. Chevalley and S. Eilenberg, Cohomology theory of Lie groups and Lie algebras, Trans. Amer. Math. Soc., vol. 63 (1948), pp. 85-124. 
3. G. Hochschild and J-P. Serre, Cohomology of group extensions, Trans. Amer. Math. Soc., vol. 74 (1953), pp. 110-134.

4. - - Cohomology of Lie algebras, Ann. of Math. (2), vol. 57 (1953), pp. 591-603.

5. G. Hochschild, Cohomology of algebraic linear groups, Illinois J. Math., vol. 5 (1961), pp. 492-519.

6. E. Kunz, Die Primidealteiler der Differenten in allgemeinen Ringen, J. Reine Angew. Math., vol. 204 (1960), pp. 165-182.

7. W. T. van Est, Une application d'une méthode de Cartan-Leray, Indag. Math., vol. 17 (1955), pp. 542-544.

\section{University of CALIFornia}

Berkeley, California 